\title{
Host Galaxies of the Highest Redshift QSOs
}

\author{
J. B. Hutchings ${ }^{1}$ \\ ${ }^{1}$ Herzberg Institute of Astrophysics, NRC of Canada, Victoria, B.C., Canada
}

Abstract. J and K-band imaging with Gemini $\mathrm{N}$ has resolved the host galaxies of QSOs of redshifts 4.7 to 5.2. We also discuss I and Z band imaging of two QSOs of redshift above 6 .

\section{Observations}

The observations were made with Gemini North with NIRI and GMOS instruments. Exposures were $\sim 2$ hours in $\mathrm{J}$ and $\mathrm{K}$ band, and $\sim 3$ hours in I and $\mathrm{Z}$ band. Image quality ranged from 0.4 " to $1.0 "$ FWHM.

The K-band data are described in Hutchings (2003). These produced resolved images of 5 QSOs at redshift 4.7 .

J-band data were obtained in Aug-Sept 2003 for two QSOs at z 5.1. These are SDSS 0338-0021, which had images of 0.43" FWHM. WFS 2245+0024 had images of FWHM $\sim 0.7$, but is about 2 magnitudes fainter, so that there is less nuclear light obscuring the host galaxy. The observations were taken with a small dither pattern and processed in a standard way. PSFs were derived from stellar images in the fields. Figures 1 and 2 show the profiles of QSO and PSF for one object. We will report in full on these results elsewhere.

The I and Z band data were taken of two QSOs of redshift above 6. These have rest wavelengths that straddle the Lyman break for the objects, so the expected host galaxy fluxes will be low (the images were taken mainly for the purpose of setting up slit masks for companion candidates). The host galaxy is marginally resolved for one, and the other has non-detection upper limits. We will report elsewhere on evidence for companions and associated structure.

\section{Discussion}

The resolved fluxes for the new objects have been extrapolated to $\mathrm{K}$ band, using mean photometric measures from $\mathrm{I}$ to $\mathrm{K}$ for the $\mathrm{z}=4.7$ QSOs, and other photometric data on these QSOs. The resulting K-z plot is shown in Figure 3. While there are likely to be significant errors in these values, the host galaxies do lie in the region that is an extension of the radio galaxies out to high redshifts, and suggest that the hosts are in a post-starburst stage of evolution, rather than being in the process of a major starburst.

There appears to be spiral structure in the central parts of these host galaxies, as well as faint extended flux over radii of several arcsec. Thus, the combination of good image quality and large collecting area is required to resolve and study host galaxies at high redshift. Deeper exposures and larger samples are required to address questions of the stellar populations and their relationship with the central black holes in these very young galaxies. 

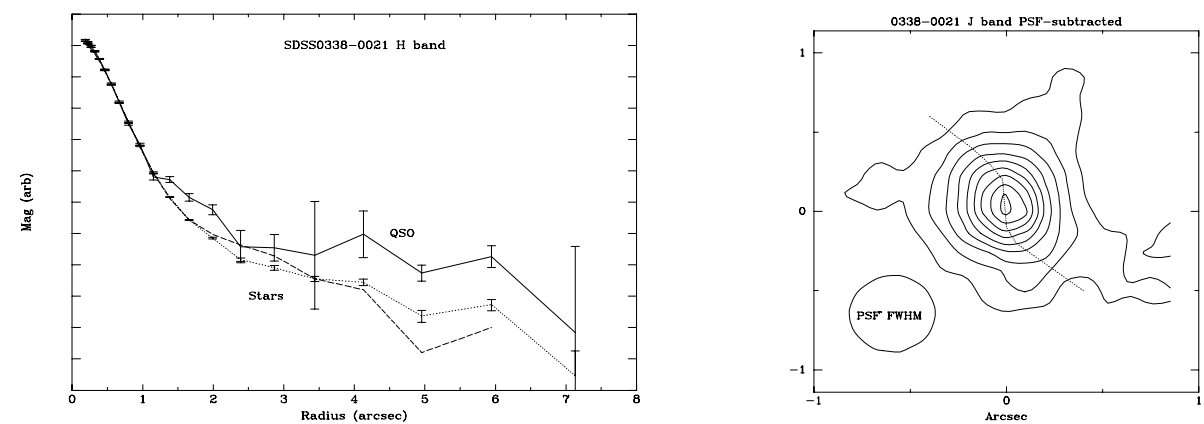

Figure 1. SDSS 0338-0021. Left: Azimuthal profile of QSO and PSFs. Right: contours of inner PSF-subtracted structure.

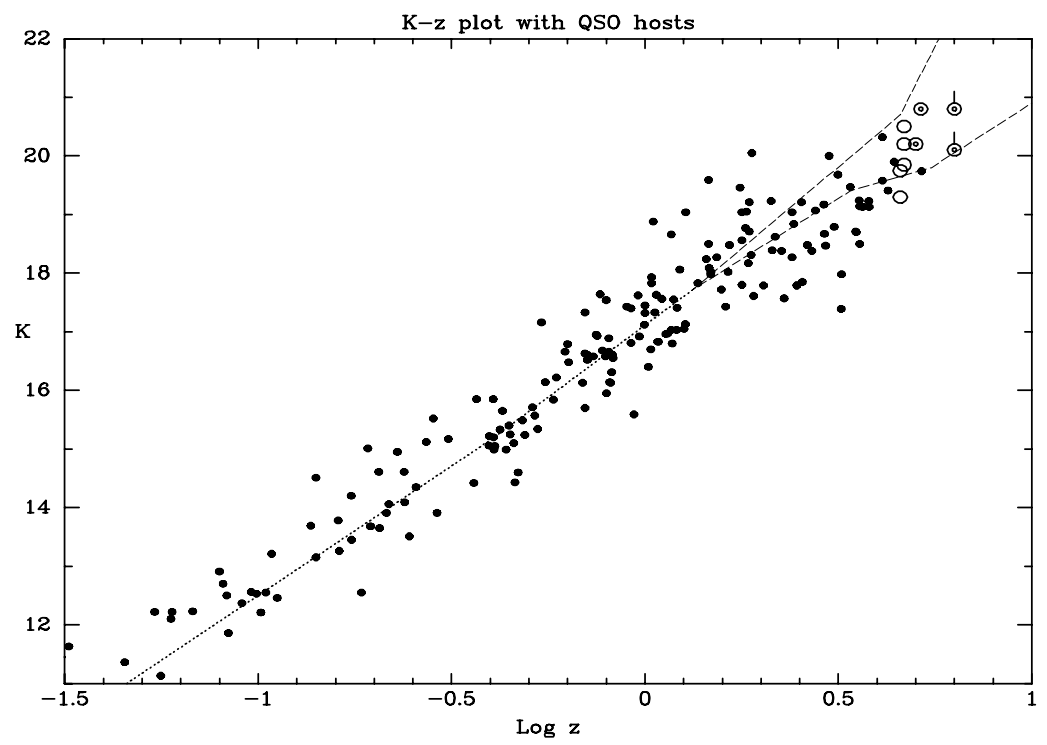

Figure 2. K-z plot for radio galaxies with high z QSO hosts

\section{References}

Hutchings, J. B. 2003, AJ, 125, 1053 